\title{
Can a routine follow-up blood culture be justified in Klebsiella pneumoniae bacteremia? a retrospective case-control study
}

\author{
Chang Kyung Kang ${ }^{1}$, Eu Suk Kim ${ }^{1,2^{*}}$, Kyoung-Ho Song ${ }^{1,2}$, Hong Bin Kim ${ }^{1,2}$, Taek Soo Kim², Nak-Hyun Kim, \\ Chung-Jong Kim 1,2, Pyoeng Gyun Choe', Ji-Hwan Bang ${ }^{1}$, Wan Beom Park', Kyoung Un Park², Sang Won Park', \\ Nam-Joong Kim ${ }^{1}$, Eui-Chong $\mathrm{Kim}^{1}$ and Myoung-don Oh${ }^{1}$
}

\begin{abstract}
Background: The need for mandatory confirmation of negative conversion in Klebsiella pneumoniae bacteremia (KpB) has not been adequately addressed. We conducted a retrospective case-control study of adult patients with $\mathrm{KpB}$ over a 5-year period in two tertiary-care hospitals to determine the risk factors for persistent bacteremia and to reevaluate the necessity of follow-up blood culture in KpB.

Methods: Persistent $K p B$ is defined as the finding of $K$. pneumoniae in more than two separate blood-culture samples for longer than a two-day period in a single episode. The case- and control-groups were patients with persistent and non-persistent $K p B$, respectively, and they were matched 1-to-3 according to age and gender.

Results: Among $1068 \mathrm{KpB}$ episodes analyzed after excluding polymicrobial infection and repeated KpB, follow-up blood cultures were performed in 862 cases (80.7\%), 62 of which (7.2\%) were persistent. Independent risk factors for persistence were intra-abdominal infection, higher Charlson's comorbidity weighted index score, prior solid organ transplantation, and unfavorable treatment response, which was defined as positivity for at least two parameters among fever, leukocytosis, and no decrease of C-reactive protein on the second day after initial culture. A proposed scoring system using four variables, namely, intra-abdominal infection, nosocomial $\mathrm{KpB}$, fever and lack of C-reactive protein decrease, the last two being assessed on the second day after the initial blood culture, showed that only $4.9 \%$ of the patients with no risk factors or with only intra-abdominal infection had persistent $\mathrm{KpB}$.
\end{abstract}

Conclusions: Though persistent KpB is uncommon, follow-up blood culture was performed in as many as $80 \%$ of the cases in this study. A more careful clinical assessment is warranted to reduce the cost and patient inconvenience involved in follow-up blood culture.

Keywords: Klebsiella pneumoniae, Bacteremia, Risk factor, Follow-up, Blood culture

\section{Background}

Klebsiella pneumoniae is one of the most important pathogens causing urinary tract infection, pneumonia, intraabdominal infection, and primary bacteremia. It is also the second most common cause of community- and hospitalacquired gram-negative bacteremia [1-3]. Mortality from Klebsiella pneumoniae bacteremia $(\mathrm{Kp} \mathrm{B})$ is about 20 to

\footnotetext{
* Correspondence: eskim@snubh.org

'Seoul National University College of Medicine, 103 Daehak-ro, Jongro-gu,

Seoul, Republic of Korea 110-460

${ }^{2}$ Department of Internal Medicine, Seoul National University Bundang

Hospital, 173-gil 82 Gumi-ro, Bundang-gu, Seongnam, Republic of Korea 463-707
}

\section{Biomed Central

(c) 2013 Kang et al.; licensee BioMed Central Ltd. This is an Open Access article distributed under the terms of the Creative Commons Attribution License (http://creativecommons.org/licenses/by/2.0), which permits unrestricted use, distribution, and reproduction in any medium, provided the original work is properly cited.

\section{1 to 9.7 per 100,000 person-years $[5,6]$.}

There is evidence that the usefulness of routine blood cultures should be reconsidered in acute pyelonephritis $[7,8]$, cellulitis [9], community-acquired pneumonia $[10,11]$, and in isolated fever or leukocytosis [12]. Although attempts have been made to reduce unnecessary blood cultures by introducing clinical rules [12-14], clear guidelines have yet to emerge as to when blood cultures should be drawn. And blood cultures are liberally prescribed because of the high mortality due to bacteremia, anxiety about undertreatment, and fear of using 
inappropriate antimicrobial agents causing limited positivity of 4 to $7 \%[12,15,16]$.

Although it is widely appreciated that the requirement for follow-up blood cultures in bacteremic patients is questionable, they may still be prescribed too liberally, resulting in low rates of positive outcomes. Although routine follow-up blood culture is recommended in Staphylococcus aureus bacteremia and in infective endocarditis [17], the need for mandatory confirmation of negative conversion in $K p \mathrm{~B}$ has not been adequately addressed. The objective of the present study was to determine the risk factors for persistent bacteremia and to reevaluate the need for routine follow-up blood cultures in $K p \mathrm{~B}$.

\section{Methods}

\section{Study setting and patients}

Data on all episodes of $K p \mathrm{~B}$ were collected from the electronic medical record systems between January 2007 and December 2011 at Seoul National University Hospital (a 1600-bed tertiary-care hospital, Seoul, Korea) and Seoul National University Bundang Hospital (a 900-bed tertiary-care hospital, Seongnam, Korea). All adult patients ( $\geq 18$ years) who suffered their first $K p \mathrm{~B}$ episodes were enrolled. Cases of polymicrobial infection, and repeated episodes of $K p \mathrm{~B}$ in the same patient, were excluded.

Case- and control-patients were those with persistent $K p \mathrm{~B}$ and non-persistent $K p \mathrm{~B}$, respectively. They were matched 1-to-3 according to age and gender using a greedy algorithm. The data reviewed included primary site of infection, Charlson's co-morbidity weighted index [18], intensive care unit (ICU) admission during the episode of $K p \mathrm{~B}$, severity of illness as measured by the Acute Physiology and Chronic Health Evaluation (APACHE) II score [19] and Pitt bacteremia score [20]; also microbiologic characteristics, both appropriateness of empirical antibiotic regimen and length of time from initial blood culture to antibiotic administration, as well as presence of fever, leukocytosis, and the trend of C-reactive protein (CRP) on the second day after initial blood culture. We also assessed the nosocomial or community onset of $K p \mathrm{~B}$, presentation with septic shock, presence of metastatic infection, antibiotic or immunosuppressive therapy within the previous year, history of solid organ transplantation, any invasive procedure undertaken and neutropenia within 72 hours before the onset of $K p \mathrm{~B}$, together with inhospital mortality, and length of hospital stay.

The study was approved by the institutional review board of Seoul National University Hospital and Seoul National University Bundang Hospital.

\section{Definitions}

The initial blood culture was defined as the first positive blood culture for $K$. pneumoniae. Persistent $K p \mathrm{~B}$ was defined as finding of $K$. pneumoniae in more than one separate blood cultures for longer than a 2-day period in a single infection episode. When an isolate was resistant to both cefotaxime and ceftazidime, it was suspected of being extended-spectrum $\beta$-lactamase (ESBL)-producing K. pneumoniae. A nosocomial infection was defined as an infection that occurred more than 48 hours after admission to hospital [21]. Fever and leukocytosis were defined as body temperature of $\geq 38^{\circ} \mathrm{C}$ and white blood cell count of $>10,000 / \mu \mathrm{L}$. Neutropenia was defined as an absolute neutrophil count below $500 / \mathrm{mL}$. Invasive procedures included insertion of a central venous catheter, endoscopy, endoscopic retrograde cholangiopancreatography, insertion of a nasogastric tube, bronchoscopy, parenteral nutrition, and insertion or manipulation of a percutaneous transhepatic biliary drainage [22].

Antimicrobial therapy was considered inappropriate if the isolate was not susceptible to the chosen antibiotic in vitro. The presence of at least two of the following: fever, leukocytosis, and lack of CRP decrease on the second day after initial blood culture, was pre-defined as an unfavorable treatment response before data collection.

\section{Microbiologic analyses}

BacT/ALERT FA and FN (bioMe'rieux, Durham, North Carolina) was used for all blood cultures. Antimicrobial susceptibility was identified with a Microscan WalkAway96 (Siemens Healthcare Diagnostics, Deerfield, Illinois) and VITEK 2 (bioMe'rieux, Marcy L'etoil, France), using the criteria of the Clinical and Laboratory Standards Institute (CLSI) guidelines. For suspected ESBL-producing K. pneumoniae isolates, ESBL production was confirmed by the double disk synergy test according to the CLSI performance standards [17].

\section{Statistical analyses}

Conditional logistic regression was used in univariable and multivariable analyses to manage matched data. Variables with $P$ values of $<0.10$ in the univariable analysis or of clinical importance (i.e., intra-abdominal infection) were subjected to multivariable analysis. To evaluate the proposed scoring system, a receiver operating characteristic (ROC) analysis was carried out. $P<0.05$ was considered statistically significant. PASW for Windows (version 18 software package; SPSS Inc., Chicago, IL, USA) was used in the analyses.

\section{Results}

Rate of follow-up blood culture and incidence of persistent $K p B$

Of a total of $1372 \mathrm{KpB}$ episodes during the study period, 1068 were included in this study, after excluding 173 cases of polymicrobial infection and 131 cases of repeated $K p \mathrm{~B}$. Among the 1068 enrolled cases of $K p \mathrm{~B}$, 
follow-up blood cultures were performed in 862 cases $(80.7 \%)$, and their incidence was highest on the second day after the initial blood culture (Additional file 1: Figure S1). Sixty-two (7.2\%) were found to be persistent $K p \mathrm{~B}$, and 186 non-persistent $K p \mathrm{~B}$ cases were matched with the latter as described above.

\section{Risk factors for persistent $K p B$}

Univariable analysis of the data indicated that the factors significantly associated with persistent $K p \mathrm{~B}$ as opposed to non-persistent $K p \mathrm{~B}$ included higher Charlson's comorbidity weighted index score, ICU admission, nosocomial infection, immunosuppressive therapy during the previous year, history of solid organ transplantation, invasive procedure during the previous 72 hours, fever and lack of CRP decrease on the second day after initial blood culture, ESBL-producing $K p \mathrm{~B}$, inappropriate empirical antibiotic use, and in-hospital mortality (Table 1).

Multivariable analysis revealed that the independent risk factors for persistent $K p \mathrm{~B}$ were intra-abdominal infection (adjusted odds ratio [aOR], 2.99; 95\% confidence interval $[\mathrm{CI}], 1.07-8.31 ; P=0.036)$, higher Charlson's

Table 1 Univariable analysis for risk factors associated with persistent Klebsiella pneumoniae bacteremia (cases) vs. non-persistent Klebsiella pneumoniae bacteremia (controls)

\begin{tabular}{|c|c|c|c|c|}
\hline Variables & Cases $(n=62)$ & Controls $(n=186)$ & OR $(95 \% \mathrm{Cl})$ & $P$ \\
\hline Age $( \pm S D)$ & $63.77( \pm 14.58)$ & $63.58( \pm 14.34)$ & & \\
\hline Male sex & $45(72.6)$ & $135(72.6)$ & & \\
\hline Urinary tract infection & $7(11.3)$ & $20(10.8)$ & $1.05(0.43-2.58)$ & 0.908 \\
\hline Intra-abdominal infection & $12(19.4)$ & $22(11.8)$ & $1.82(0.83-4.00)$ & 0.136 \\
\hline Biliary tract infection & $13(21.0)$ & $53(28.5)$ & $0.65(.032-1.32)$ & 0.236 \\
\hline Liver abscess & $8(12.9)$ & $31(16.7)$ & $0.73(0.31-1.72)$ & 0.469 \\
\hline Pneumonia & $8(12.9)$ & $18(9.7)$ & $1.36(0.57-3.20)$ & 0.487 \\
\hline Primary bacteremia & $9(14.5)$ & $34(18.3)$ & $0.73(0.31-1.72)$ & 0.474 \\
\hline Central venous catheter related infection & $2(3.2)$ & $4(2.2)$ & $1.50(0.28-8.19)$ & 0.640 \\
\hline Charlson's CWI score ( \pm SD) & $6.29( \pm 3.05)$ & $5.52( \pm 2.94)$ & $1.14(1.01-1.28)$ & 0.037 \\
\hline ICU admission & $18(29.0)$ & $30(16.1)$ & $2.12(1.08-4.16)$ & 0.029 \\
\hline Presentation with septic shock & $22(35.5)$ & $53(28.5)$ & $1.35(0.75-2.44)$ & 0.316 \\
\hline APACHE-II score $( \pm S D)$ & $18.97( \pm 9.28)$ & $17.09( \pm 8.16)$ & $1.03(0.99-1.06)$ & 0.140 \\
\hline Pitt bacteremia score $( \pm \mathrm{SD})$ & $2.45( \pm 2.67)$ & $2.17( \pm 2.45)$ & $1.04(0.94-1.16)$ & 0.452 \\
\hline Nosocomial infection & $34(54.8)$ & $55(29.6)$ & $2.96(1.61-5.43)$ & $<0.001$ \\
\hline Previous antibiotic use $\leq 1$ year & $37(59.7)$ & $95(51.1)$ & $1.42(0.79-2.54)$ & 0.240 \\
\hline Immunosuppressive therapy $\leq 1$ year & $5(8.1)$ & $4(2.2)$ & $4.46(1.05-18.95)$ & 0.043 \\
\hline History of solid organ transplantation & $4(6.5)$ & $2(1.1)$ & $10.18(1.11-93.02)$ & 0.040 \\
\hline Invasive procedure $\leq \mathbf{7 2}$ hours before initial $\mathrm{BC}$ & $20(32.3)$ & $30(16.1)$ & $2.56(1.29-5.07)$ & 0.007 \\
\hline Neutropenia $\leq 72$ hours before initial BC & $11(17.7)$ & $31(16.7)$ & $1.08(0.50-2.33)$ & 0.843 \\
\hline Presence of metastatic infection & $5(8.1)$ & $9(4.8)$ & $1.67(0.56-4.97)$ & 0.360 \\
\hline Inappropriate drainage & $11(17.7)$ & $20(10.8)$ & $1.81(0.80-4.07)$ & 0.154 \\
\hline Fever on the $2^{\text {nd }}$ day after initial $B C$ & $38(61.3)$ & $46(24.7)$ & $5.18(2.64-10.16)$ & $<0.001$ \\
\hline Leukocytosis on the $2^{\text {nd }}$ day after initial $B C$ & $23(37.1)$ & $60(32.3)$ & $1.22(0.68-2.19)$ & 0.499 \\
\hline Lack of CRP decrease on the $2^{\text {nd }}$ day after initial BC & $26(41.9)$ & $43(23.1)$ & $2.38(1.29-4.39)$ & 0.010 \\
\hline Unfavorable treatment response on the $2^{\text {nd }}$ day after initial $B C$ & $27(43.5)$ & $30(16.1)$ & $4.69(2.27-9.69)$ & $<0.001$ \\
\hline ESBL-producing Klebsiella pneumoniae bacteremia & $28(45.2)$ & $31(16.7)$ & $3.99(2.09-7.63)$ & $<0.001$ \\
\hline Inappropriate empirical antibiotic use & $22(35.5)$ & $24(12.9)$ & $4.02(1.95-8.31)$ & $<0.001$ \\
\hline Length of time to empirical antibiotic administration from initial BC (minute, \pm SD) & $182.8( \pm 221.3)$ & $228.4( \pm 434.8)$ & $1.00(0.99-1.00)$ & 0.430 \\
\hline In-hospital mortality & 19 (30.6) & $34(18.3)$ & $1.96(1.02-3.78)$ & 0.044 \\
\hline Length of hospital stay (day, \pm SD) & $45.1( \pm 43.0)$ & $38.6( \pm 74.9)$ & $1.00(0.99-1.01)$ & 0.524 \\
\hline
\end{tabular}

Data are no. (\%) of patients, unless otherwise indicated.

$\mathrm{OR}$, odds ratio; $\mathrm{Cl}$, confidence interval; $\mathrm{SD}$, standard deviation; $\mathrm{CWl}$, comorbidity weighted index; ICU, intensive care unit; APACHE, acute physiology and chronic health evaluation; $B C$, blood culture; $C R P, C$-reactive protein; ESBL, extended-spectrum $\beta$-lactamase. 
comorbidity weighted index score (aOR, 1.18 per each point; 95\% CI, 1.01-1.36; $P=0.032$ ), history of solid organ transplantation (aOR, 92.23; 95\% CI, 1.27-6689.96; $P=0.038$ ), and unfavorable treatment response (aOR, 4.79; 95\% CI, 1.89-12.14; $P=0.001)$. The concept of unfavorable treatment response was introduced and substituted for fever, leukocytosis, and lack of CRP decrease in the multivariable analysis, to avoid the multicolinearity among those three variables. Neither ESBL-producing $K p B$ nor inappropriate empirical antibiotic use was found to be an independent risk factor (Table 2).

\section{Percentage of persistent $K p B$ according to clinical score}

To estimate the likelihood of persistent $K p \mathrm{~B}$, a scoring system was introduced, based on the presence or absence of four risk factors viz. intra-abdominal infection, nosocomial $K p B$, fever and lack of CRP decrease, the last two being assessed on the second day after initial blood culture. According to their odds ratio in a conditional logistic regression with these four risk factors, the weighted scores given for the risk factors were 1, 2, 3, and 2 , respectively (Table 3 ).

The frequency of persistent $K p \mathrm{~B}$ exceeded $50 \%$ when the score was greater than five; conversely, it was only $4.9 \%$ when the score was zero to one with no risk factors, or with intra-abdominal infection as the only risk factor (Figure 1). This indicates that persistent $K p \mathrm{~B}$ is likely to be rarer among patients with fewer risk factors. A ROC analysis showed that the area under the curve was $0.78(P<0.0001)$ and the best cut-off was 2 (sensitivity, 0.81; specificity, 0.64, Additional file 2: Figure S2).

\section{Discussion}

K. pneumoniae is one of the most important pathogens causing nosocomial and community-acquired infections, and clinicians sometimes encounter persistent $K p \mathrm{~B}$ in clinical practice. Despite the fact that the CLSI guidelines do not recommend routine follow-up blood cultures except in cases of Staphylococcus aureus bacteremia and infective endocarditis [17], our understanding of the occurrence of persistent $K p \mathrm{~B}$ and the frequency of follow-up blood cultures is far from complete. The present study showed that, although follow-up blood cultures were performed in $80.7 \%$ of the cases, only $7.2 \%$ were found to be persistent $K p B$. Furthermore, more than two consecutive blood cultures were performed in $53.2 \%$ of the patients with non-persistent $K p \mathrm{~B}$ in the present study (Data not shown). These results imply that most of these followup blood cultures would have been unwarranted. Studies have shown that only 4 to $7 \%$ of blood cultures are positive owing to its liberal prescription [12,15,16], and our data with follow-up blood cultures also point to a similar situation. Our multivariable analysis revealed that unfavorable treatment response was an independent risk factor for persistent $K p \mathrm{~B}$, along with intra-abdominal infection, high Charlson's comorbidity weighted index score, and prior solid organ transplantation. All this suggests that, when treating suspected $K p \mathrm{~B}$ patients, the clinical findings should be considered carefully before opting for follow-up blood culture, to reduce indiscriminate prescription and the ensuing cost and patient inconvenience.

Severe underlying disease and prior organ transplantation are established risk factors for $K p \mathrm{~B}[4,5,23,24]$, as they were for persistent $K p \mathrm{~B}$ in this study. An intraabdominal origin of $K p \mathrm{~B}$ is a known risk factor for mortality $[4,5]$, and it was also significantly associated with persistent $K p \mathrm{~B}$ in our analysis. Although the $K p \mathrm{~B}$ of respiratory tract origin has been documented to be a risk factor for mortality $[4,5,23]$, it was not significantly associated with persistent $K p \mathrm{~B}$ in this study. It would be interesting to find their association further by taking many factors into account, including the limitations of this study as explained below.

Table 2 Independent risk factors for persistent Klebsiella pneumoniae bacteremia

\begin{tabular}{|c|c|c|}
\hline Variables & Adjusted OR $(95 \% \mathrm{Cl})$ & $P$ \\
\hline Intra-abdominal infection & $2.99(1.07-8.31)$ & 0.036 \\
\hline Charlson's CWI score ( \pm SD) & $1.18(1.01-1.36)$ & 0.032 \\
\hline ICU admission & $1.98(0.80-4.87)$ & 0.139 \\
\hline Nosocomial infection & $2.05(0.81-5.21)$ & 0.130 \\
\hline Immunosuppressive therapy $\leq 1$ year & $0.36(0.02-8.90)$ & 0.533 \\
\hline History of solid organ transplantation & $92.23(1.27-6689.96)$ & 0.038 \\
\hline Invasive procedure $\leq 72$ hours before initial BC & $0.77(0.27-2.19)$ & 0.618 \\
\hline Unfavorable treatment response on the $2^{\text {nd }}$ day after initial $B C$ & $4.79(1.89-12.14)$ & 0.001 \\
\hline ESBL-producing Klebsiella pneumoniae bacteremia & $1.72(0.63-4.69)$ & 0.293 \\
\hline Inappropriate empirical antibiotic use & $2.76(0.93-8.23)$ & 0.068 \\
\hline
\end{tabular}

$\mathrm{OR}$, odds ratio; $\mathrm{Cl}$, confidence interval; $\mathrm{SD}$, standard deviation; $\mathrm{CWI}$, comorbidity weighted index; ICU, intensive care unit; $\mathrm{BC}$, blood culture; $\mathrm{ESBL}$, extended-spectrum $\beta$-lactamase. 
Table 3 Odds ratios and adjusted weighted scores for the four variables used in the scoring system

\begin{tabular}{|c|c|c|c|}
\hline Variables & OR & $\log (\mathrm{OR})$ & Adjusted weighted score \\
\hline Intra-abdominal infection & 1.87 & 0.63 & 1 \\
\hline Nosocomial infection & 3.24 & 1.18 & 2 \\
\hline Fever on the $2^{\text {nd }}$ day after initial blood culture & 8.30 & 2.12 & 3 \\
\hline Lack of CRP decrease on the $2^{\text {nd }}$ day after initial blood culture & 3.10 & 1.13 & 2 \\
\hline
\end{tabular}

$\mathrm{OR}$, odds ratio; CRP, C-reactive protein.

According to Kang et al. [25,26], ESBL-producing organism and inappropriate empirical antibiotic are not risk factors for increased mortality in bloodstream infections due to K. pneumoniae. We also found that production of ESBL was not an independent risk factor for persistent $K p \mathrm{~B}$, which indicates that follow-up blood cultures are not routinely needed in cases of ESBLproducing $K p$ B. However, inappropriate empirical antibiotic regimen may call for further evaluation because of its borderline $P$ value.

Various definitions of persistent bacteremia have been used in Staphylococcus aureus bacteremia [27-29]. Our definition of persistent bacteremia was not as strict as others because both the failure of appropriate antimicrobial therapy and prolonged periods of bacteremia were not essential elements of our consideration. If a stricter definition had been used, the incidence of persistent $K p \mathrm{~B}$ would have been further reduced, which supports our suggestion that more care should be taken before opting for follow-up blood culture.

Our scoring system, which has fair discriminative ability, could be used as a guide for whether a follow-up blood culture is needed, since there was a positive relationship between clinical score and percent persistent $K p \mathrm{~B}$ (Figure 1): the percentage of persistent $K p \mathrm{~B}$ declined sharply as the score decreased, and less than $5 \%$ of the patients with zero or one point had persistent bacteremia. On the other hand, follow-up blood culture could be justified in patients with more than five points because more than a half of those would be expected to suffer from persistent bacteremia. According to our data, if follow-up blood cultures were only carried out in patients with scores exceeding 2 points, more than $50 \%$ of follow-up blood cultures could be avoided (Figure 1).

There are some limitations to this study. There is a possibility of bias because it was a retrospective study with a limited sample size. Although our scoring system appeared to be useful, as discussed above, it should be further assessed on a larger sample. We were not able to evaluate the possible impact of age and gender on persistent $K p \mathrm{~B}$ by matching for these factors because of the design of this study. Also, there may have been selection bias in the $19.3 \%$ of patients without follow-up blood culture, one third of whom died by the 2nd day after blood culture (data not shown).

Persistent $K p \mathrm{~B}$ showed significantly worse outcomes, such as in-hospital mortality $(P=0.044)$ and ICU admission during the $K p \mathrm{~B}$ episode $(P=0.029)$ in univariable analyses (Table 1 ). However, we did not perform multivariable analysis for mortality-related factors, mainly

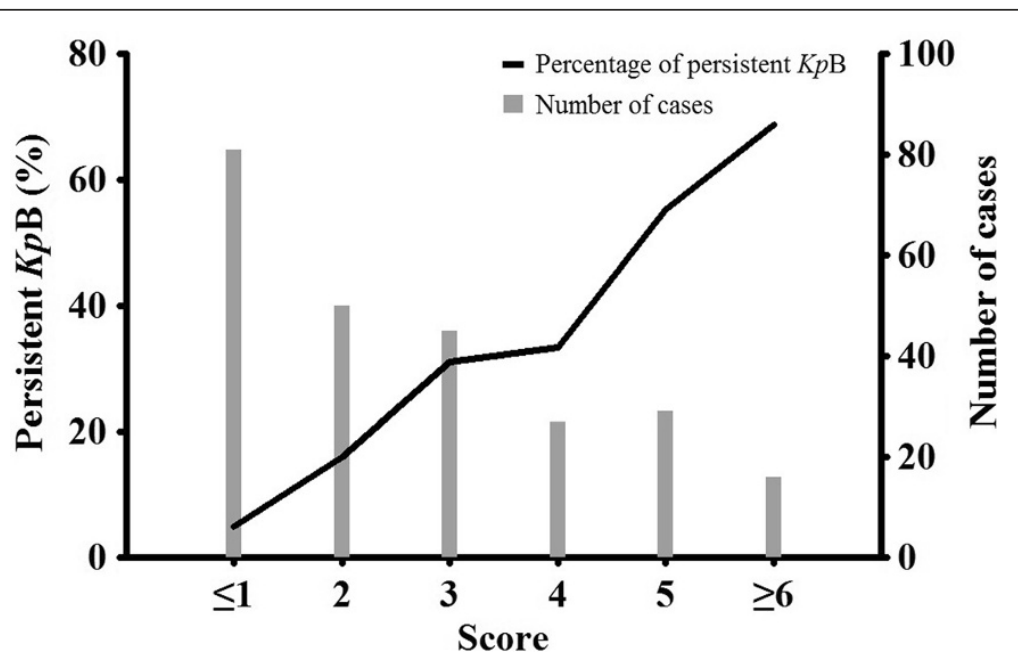

Figure 1 Percentage of persistent Klebsiella pneumoniae bacteremia and number of cases according to clinical score. There was a positive relationship between clinical score and percent persistent $\mathrm{KpB}$. 
because the study design was focused on persistence of bacteremia, and also the matching was carried out in that context.

Finally, this study was undertaken in universityaffiliated tertiary-care hospitals where a large proportion of the patients might have severe underlying diseases including malignancy. However, $64.1 \%$ and $25.0 \%$ of our cases involved community-onset and communityacquired $K p \mathrm{~B}$, respectively (data not shown). The rate of persistent $K p \mathrm{~B}$ would be expected to be much lower in a community-based hospital since a high Charlson's comorbidity weighed index score is an independent risk factor for persistent $K p \mathrm{~B}$ and the comorbidities in patients in community-based hospitals are likely to be less severe.

\section{Conclusions}

In conclusion, unfavorable treatment response on the second day after initial blood culture, intra-abdominal infection, high Charlson's comorbidity weighted index score, and prior solid organ transplantation are independent risk factors for persistent $K p$ B. Since patients with persistent $K p \mathrm{~B}$ were rare, especially among individuals with few risk factors, routine follow-up blood culture may not be justified. More careful clinical assessment before deciding on follow-up blood culture would reduce costs and inconvenience to patients.

\section{Additional files}

Additional file 1: Figure S1. Numbers of follow-up blood cultures, and numbers of those cultures positive for Klebsiella pneumoniae among the total of 1068 patients, according to days after the initial blood cultures.

Additional file 2: Figure S2. Receiver operating characteristic analysis of the proposed clinical scoring system.

\section{Competing interests}

The authors declare that they have no competing interests.

\section{Authors' contributions}

CKK, ESK, KHS, and HBK participated in the study design, data extraction, analysis of data, and writing of the manuscript. TSK, KUP, and ECK participated in data collection. NHK, CJK, PGC, JHB, WBP, SWP, NJK, and MDO advised on analysis and interpretation. All authors read and approved the final manuscript.

Received: 23 March 2013 Accepted: 25 July 2013

Published: 2 August 2013

\section{References}

1. Skogberg K, Lyytikainen O, Ruutu P, Ollgren J, Nuorti JP: Increase in bloodstream infections in Finland, 1995-2002. Epidemiol Infect 2008, 136(1):108-114.

2. Uslan DZ, Crane SJ, Steckelberg JM, Cockerill FR 3rd, St Sauver JL, Wilson WR, Baddour LM: Age- and sex-associated trends in bloodstream infection: a population-based study in Olmsted County, Minnesota. Arch Intern Med 2007, 167(8):834-839.

3. Yinnon AM, Butnaru A, Raveh D, Jerassy Z, Rudensky B: Klebsiella bacteraemia: community versus nosocomial infection. QJM 1996, 89(12):933-941.

4. Kang Cl, Kim SH, Bang JW, Kim HB, Kim NJ, Kim EC, Oh MD, Choe KW: Community-acquired versus nosocomial Klebsiella pneumoniae bacteremia: clinical features, treatment outcomes, and clinical implication of antimicrobial resistance. J Korean Med Sci 2006, 21(5):816-822.

5. Meatherall BL, Gregson D, Ross T, Pitout JD, Laupland KB: Incidence, risk factors, and outcomes of Klebsiella pneumoniae bacteremia. Am J Med 2009, 122(9):866-873.

6. Al-Hasan MN, Lahr BD, Eckel-Passow JE, Baddour LM: Epidemiology and outcome of Klebsiella species bloodstream infection: a population-based study. Mayo Clin Proc 2010, 85(2):139-144.

7. Velasco M, Martinez JA, Moreno-Martinez A, Horcajada JP, Ruiz J, Barranco M, Almela M, Vila J, Mensa J: Blood cultures for women with uncomplicated acute pyelonephritis: are they necessary? Clin Infect Dis 2003, 37(8):1127-1130.

8. Chen Y, Nitzan O, Saliba W, Chazan B, Colodner R, Raz R: Are blood cultures necessary in the management of women with complicated pyelonephritis? J Infect 2006, 53(4):235-240.

9. Paolo WF, Poreda AR, Grant W, Scordino D, Wojcik S: Blood culture results do not affect treatment in complicated cellulitis. J Emerg Med 2013. Apr 12. [Epub ahead of print].

10. Campbell SG, Marrie TJ, Anstey R, Dickinson G, Ackroyd-Stolarz S: The contribution of blood cultures to the clinical management of adult patients admitted to the hospital with community-acquired pneumonia: a prospective observational study. Chest 2003, 123(4):1142-1150.

11. Afshar N, Tabas J, Afshar K, Silbergleit R: Blood cultures for communityacquired pneumonia: are they worthy of two quality measures? A systematic review. J Hosp Med 2009, 4(2):112-123.

12. Coburn B, Morris AM, Tomlinson G, Detsky AS: Does this adult patient with suspected bacteremia require blood cultures? JAMA 2012, 308(5):502-511.

13. Shapiro NI, Wolfe RE, Wright SB, Moore R, Bates DW: Who needs a blood culture? A prospectively derived and validated prediction rule. J Emerg Med 2008, 35(3):255-264.

14. Jones GR, Lowes JA: The systemic inflammatory response syndrome as a predictor of bacteraemia and outcome from sepsis. QJM 1996, 89(7):515-522

15. Bates DW, Cook EF, Goldman L, Lee TH: Predicting bacteremia in hospitalized patients. A prospectively validated model. Ann Intern Med 1990, 113(7):495-500.

16. Roth A, Wiklund AE, Palsson AS, Melander EZ, Wullt M, Crongvist J, Walder M, Sturegard E: Reducing blood culture contamination by a simple informational intervention. J Clin Microbiol 2010, 48(12):4552-4558.

17. Clinical and Laboratory Standards Institute: Principles and procedures for blood cultures; approved guideline. CLSI document M47-A. Wayne, PA: Clinical and Laboratory Standards Institute; 2007

18. Charlson ME, Pompei P, Ales KL, MacKenzie CR: A new method of classifying prognostic comorbidity in longitudinal studies: development and validation. J Chronic Dis 1987, 40(5):373-383.

19. Knaus WA, Draper EA, Wagner DP, Zimmerman JE: APACHE II: a severity of disease classification system. Crit Care Med 1985, 13(10):818-829.

20. Chow JW, Fine MJ, Shlaes DM, Quinn JP, Hooper DC, Johnson MP, Ramphal R, Wagener MM, Miyashiro DK, Yu VL: Enterobacter bacteremia: clinical features and emergence of antibiotic resistance during therapy. Ann Intern Med 1991, 115(8):585-590.

21. Garner JS, Jarvis WR, Emori TG, Horan TC, Hughes JM: CDC definitions for nosocomial infections, 1988. Am J Infect Control 1988, 16(3):128-140.

22. Tumbarello M, Spanu T, Sanguinetti M, Citton R, Montuori E, Leone F, Fadda G, Cauda R: Bloodstream infections caused by extended-spectrum-betalactamase-producing Klebsiella pneumoniae: risk factors, molecular epidemiology, and clinical outcome. Antimicrob Agents Chemother 2006, 50(2):498-504

23. Tsay RW, Siu LK, Fung CP, Chang FY: Characteristics of bacteremia between community-acquired and nosocomial Klebsiella pneumoniae infection: risk factor for mortality and the impact of capsular serotypes as a herald for community-acquired infection. Arch Intern Med 2002, 162(9):1021-1027.

24. Wang LS, Lee FY, Cheng DL, Liu CY, Hinthorn DR, Jost PM: Klebsiella pneumoniae bacteremia: analysis of 100 episodes. J Formos Med Assoc 1990, 89(9):756-763.

25. Kang Cl, Kim SH, Kim DM, Park WB, Lee KD, Kim HB, Oh MD, Kim EC, Choe KW: Risk factors for and clinical outcomes of bloodstream infections caused by extended-spectrum beta-lactamase-producing Klebsiella pneumoniae. Infect Control Hosp Epidemiol 2004, 25(10):860-867. 
26. Kang Cl, Kim SH, Park WB, Lee KD, Kim HB, Kim EC, Oh MD, Choe KW: Bloodstream infections due to extended-spectrum beta-lactamase -producing Escherichia coli and Klebsiella pneumoniae: risk factors for mortality and treatment outcome, with special emphasis on antimicrobial therapy. Antimicrob Agents Chemother 2004, 48(12):4574-4581.

27. Hawkins C, Huang J, Jin N, Noskin GA, Zembower TR, Bolon M: Persistent Staphylococcus aureus bacteremia: an analysis of risk factors and outcomes. Arch Intern Med 2007, 167(17):1861-1867.

28. Fowler VG Jr, Sakoulas G, McIntyre LM, Meka VG, Arbeit RD, Cabell CH, Stryjewski ME, Eliopoulos GM, Reller LB, Corey GR, et al: Persistent bacteremia due to methicillin-resistant Staphylococcus aureus infection is associated with agr dysfunction and low-level in vitro resistance to thrombin-induced platelet microbicidal protein. J Infect Dis 2004, 190(6):1140-1149.

29. Jang HC, Kim SH, Kim KH, Kim CJ, Lee S, Song KH, Jeon JH, Park WB, Kim $H B$, Park SW, et al: Salvage treatment for persistent methicillin-resistant Staphylococcus aureus bacteremia: efficacy of linezolid with or without carbapenem. Clin Infect Dis 2009, 49(3):395-401.

doi:10.1186/1471-2334-13-365

Cite this article as: Kang et al: Can a routine follow-up blood culture be justified in Klebsiella pneumoniae bacteremia?

a retrospective case-control study. BMC Infectious Diseases 2013 13:365.

\section{Submit your next manuscript to BioMed Central and take full advantage of:}

- Convenient online submission

- Thorough peer review

- No space constraints or color figure charges

- Immediate publication on acceptance

- Inclusion in PubMed, CAS, Scopus and Google Scholar

- Research which is freely available for redistribution 